\title{
Características clínicas y administrativas que se relacionan con el retraso en el diagnóstico y tratamiento de los pacientes con cáncer de cabeza y cuello
}

\author{
Clinical and administrative characteristics related to the delay in the \\ diagnosis and treatment of patients with head and neck cancer
}

\author{
Andrés Felipe López ${ }^{1}$ Álvaro Enrique Sanabria²
}

\begin{abstract}
Residente de Cirugía General, Departamento de Cirugía, Universidad de Antioquia, Medellín, Colombia
Profesor, Departamento de Cirugía, Universidad de Antioquia; cirujano de cabeza y cuello, Fundación Colombiana de Cancerología Clínica Vida, Medellín, Colombia
\end{abstract}

\section{Resumen}

Introducción. En Colombia, el diagnóstico de los tumores de cabeza y cuello es tardío, lo cual aumenta la mortalidad, las secuelas estéticas y funcionales, y los costos para el sistema de salud. Actualmente, hay poca información sobre los factores que retrasan el diagnóstico y tratamiento de los pacientes con cáncer de cabeza y cuello.

Métodos. Se trata un estudio transversal y analítico. Como referencia, se utilizó el cálculo de muestras para una población finita con un nivel de confianza del $95 \%$ y un margen de error del $5 \%$, para un total de 159 pacientes con diagnóstico de cáncer de cabeza y cuello atendidos en la Clínica Vida de Medellín, entre los años 20I6 y 2017.

Resultados. Se evaluaron I46 pacientes. Los síntomas más frecuentes por los que consultaron fueron masa cervical y disfonía. Solo el 53,4 \% de los pacientes buscó atención médica inmediata ante la presencia del síntoma. De aquellos que no la buscaron, la causa más frecuente fue la creencia de que el síntoma desaparecería espontáneamente.

Discusión. Con base en los resultados obtenidos, se puede concluir que el síntoma de presentación juega un papel importante para que el paciente decida buscar o no buscar atención médica inmediata. El síntoma de presentación y el vivir en el área rural, retrasan el tiempo transcurrido entre la aparición del síntoma y la primera valoración por un médico de atención primaria. Los factores que se asociaron al retraso entre el diagnóstico y el inicio del tratamiento, fueron la seguridad social y el haber presentado tos como síntoma inicial.

Palabras clave: neoplasias de cabeza y cuello; diagnóstico tardío; tiempo de tratamiento; política pública; factores epidemiológicos; factores determinantes sociales de la salud.

Fecha de recibido: 30/06/2019 - Fecha aceptación: 21/08/2019

Correspondencia: Andrés Felipe López, Carrera 80 N³ 34-B-61, Medellín, Colombia. Teléfono: (319) 360-8374

Correo electrónico: pipelopez1985@gmail.com

Citar como: López AF, Sanabria AE. Características clínicas y administrativas que se relacionan con el retraso en el diagnóstico y tratamiento de los pacientes con cáncer de cabeza y cuello. Rev Colomb Cir. 2020;35:66-74. https://doi.org/10.30944/20117582.589

Este es un artículo de acceso abierto bajo una Licencia Creative Commons - BY-NC-ND https://creativecommons.org/licenses/by-ncnd/4.0/deed.es 


\begin{abstract}
Introduction: In Colombia the diagnosis of head and neck tumors is late, which represents an increase in mortality, aesthetic and functional sequelae and costs for the health system. Currently, there is little information on the factors that delay the diagnosis and treatment of patients with head and neck cancer.

Methods: It is a cross-sectional and analytical study. As a reference, the calculation of samples was used for a finite population with a confidence level of $95 \%$ and a margin of error of $5 \%$, for a total of 159 patients diagnosed with head and neck cancer treated between 2016 and 2017.

Results: A total of I46 patients were evaluated. The most frequent symptoms for those who consulted were neck mass and dysphonia. Only $53.4 \%$ of the patients sought immediate medical attention in the presence of the symptom. Of those who did not seek for it, the most frequent cause was the belief that the symptom would spontaneously disappear.

Discussion: Based on the results obtained, it can be concluded that the presentation symptom plays an important role for the patient to decide whether or not to seek immediate medical attention. The presentation symptom and living in rural areas delay the time elapsed between the appearance of the symptom and the first assessment by a primary care physician. The factors that were associated with the delay between diagnosis and the start of treatment were social security and having presented cough as an initial symptom.
\end{abstract}

Keywords: head and neck neoplasms; delayed diagnosis; time-to-treatment; public policy; epidemiologic factors ; social determinants of health.

\section{Introducción}

Los tumores malignos de cabeza y cuello representan, en conjunto, el sexto cáncer más común y corresponden del 3 o al $4 \%$ de todos los tumores en Estados Unidos y Europa, respectivamente, con una incidencia aproximada de 500.000 casos cada año y una mortalidad global cercana al $50 \%$ '. De 2007 a 20II, en Colombia, se presentaron, 62.818 cánceres, de los cuales I.494 correspondieron a cáncer de labio, cavidad oral y faringe, sin que existiera una predilección según el sexo, con un total de 787 hombres y 707 mujeres ${ }^{2}$.

Los principales factores de riesgo son el consumo de tabaco y alcohol, aunque en los últimos años se ha encontrado una importante relación entre el virus de papiloma humano y los cánceres de cabeza y cuello, principalmente aquellos ubicados en la base de la lengua y las amígdalas, relación aún mayor en hombres que no consumen alcohol ni tabaco ${ }^{3,4}$.

En Colombia, el diagnóstico de los tumores de cabeza y cuello es tardío. Se sabe que el diagnóstico tardío o el retraso en el inicio del tratamiento conllevan aumento en la morbimortalidad de estos pacientes, con una disminución en la supervivencia de 70 a $90 \%$ a los cinco años en los estadios I y II, que disminuye de 20 a $55 \%$ en los estadios avanzados 5 .

Debido a que se desconoce la razón del retraso en el diagnóstico, se decidió realizar el presente estudio, con el fin de que la identificación de esos factores pueda ser utilizada posteriormente por las autoridades sanitarias de Colombia para emprender campañas que disminuyan los tiempos en el diagnóstico y tratamiento de estos pacientes, que incluirían la educación poblacional sobre factores de riesgo, la identificación de signos de alarma para buscar atención oportuna, la educación del médico de atención primaria, la búsqueda activa de la enfermedad en pacientes con factores de riesgo, y la agilización de los procesos de remisión y los exámenes paraclínicos.

Entre las posibles variables que podrían estar relacionadas con este retraso, se encuentra que el paciente considere de poca importancia o menosprecie los síntomas al inicio de su enfermedad y, por ende, no consulte al médico de 
atención primaria de forma oportuna, que no confíe en la medicina tradicional o que no cuente con seguro de salud. En este último caso, vale la pena mencionar que, en Colombia, alrededor de 7 millones de personas son pobres y, de estas, 2 millones son indigentes. Mientras que el $30 \%$ de la población urbana en Colombia es pobre, en áreas rurales la cifra se eleva al $65 \%$, por lo que las condiciones socioeconómicas o el lugar de procedencia podrían ser un obstáculo para acceder a una atención oportuna ${ }^{6}$.

En Colombia, existen entidades promotoras de salud (EPS), las cuales son aseguradoras encargadas de administrar y gestionar todo lo referente a la salud de los individuos, como cubrimiento de los gastos de atención, contratación con hospitales para la atención y el tratamiento de los pacientes, y administración de medicamentos; por esto, en determinado caso, una mala gestión por parte de las EPS implica que al paciente se le retrasen las citas con el especialista, la práctica de un examen diagnóstico o el inicio de un tratamiento.

Existe también un recurso judicial, conocido como acción de tutela, a la cual se puede acudir en caso de que se considere que existe una vulneración del derecho a la salud; por ende, se utilizó el uso o no uso de este recurso para medir el impacto de las aseguradoras en un posible retraso de la atención, el diagnóstico o el tratamiento.

Por último, por su especial ubicación anatómica, los tumores de cabeza y cuello requieren tratamientos que pueden ser de gran morbilidad en muchos casos, la cual incluye secuelas estéticas no satisfactorias, xerostomía, necesidad de traqueostomía, gastrostomías y pérdida de la voz, entre otras. Por todo esto, en esta investigación se pretendió determinar cuáles son los factores clínicos y administrativos relacionados con un retraso en el diagnóstico y el tratamiento oportunos de los pacientes con los tumores malignos de la vía aerodigestiva superior.

\section{Métodos}

Este es un estudio descriptivo y transversal, y fue aprobado con el Comité de Ética en Investigación de la Fundación Colombiana de Cancerología. La población blanco fueron aquellos pacientes adultos con cáncer de cabeza y cuello atendidos por la especialidad de cirugía de cabeza y cuello en cualquier estadio o fase de tratamiento. Se incluyeron los pacientes adultos con cáncer de cabeza y cuello en cualquier estadio o fase de tratamiento, atendidos por el servicio de cirugía de cabeza y cuello de la Fundación Colombiana de Cancerología Clínica Vida (Medellín), según los criterios de inclusión y exclusión.

El tamaño de la muestra se calculó en I59 pacientes con un nivel de confianza del $95 \%$ y un margen de error del $5 \%$, a partir de una muestra global de los 258 atendidos en un año.

De acuerdo con la Resolución oo8430 de 1993 del Ministerio de Salud de Colombia, se consideró que la investigación no tenía riesgo para los pacientes y, por lo tanto, no se diligenció el consentimiento informado.

La información obtenida se presenta en gráficos y tablas de frecuencias. Las variables categóricas se presentan como proporciones y, las variables continuas, como promedio y desviación estándar o medianas. La comparación entre las variables categóricas se realizó con la prueba de ji al cuadrado o el test exacto de Fisher. La comparación entre las variables continuas con distribución normal, se hizo mediante la prueba t de Student y se utilizó la prueba de Mann-Whitney para las variables con distribución diferente a la normal. La normalidad de las variables se calculó con la prueba de Shapiro-Wilk.

Se calculó la razón de momios (Odds Ratio, OR) con un intervalo de confianza del $95 \%$, utilizando una tabla de $2 \times 2$. Los cálculos se hicieron con el programa Stata $9.0^{\mathrm{TM}}$. Se realizó un análisis de regresión logística exploratorio para los resultados de retraso, incluyendo los factores que se consideraron clínicamente relevantes.

\section{Resultados}

Finalmente, se incluyeron en el estudio I46 pacientes en total. Entre las características demográficas de la población, encontramos: una edad promedio de 64,5 \pm II,2 años (mediana: 66; rango: 
3I-88); el 64,4 \% fueron hombres; el 98,6 \% pertenecía a un nivel socioeconómico medio o bajo; el $27 \%$ era desempleado, y el $78,8 \%$ tuvo educación básica. La mayoría de los pacientes pertenecía al régimen contributivo y vivía en una zona urbana.

El 97,9\% de los pacientes presentó carcinoma escamocelular y las localizaciones más frecuentes fueron la laringe y la cavidad oral. El 59,6 \% consultó en los estadios III y IV de la enfermedad, y el tratamiento más frecuente fue la cirugía seguida de quimioterapia. Las características demográficas clínicas se presentan en la tabla I.

Los síntomas más frecuentes por los que consultaron los pacientes, fueron la presencia de masa cervical y la disfonía (tabla 2). Solo el $53,4 \%$ de los pacientes buscó atención médica inmediata ante la presencia del síntoma y, en los demás, la causa más frecuente para no hacerlo fue la creencia de que el síntoma desaparecería espontáneamente.

El 63,3\% de los pacientes consideraron el síntoma de nula o mínima gravedad, y el 4,8 \% optó por un tratamiento casero. Los intervalos de tiempo analizados mayores de dos meses, fueron: desde el primer síntoma hasta la revisión por un médico de atención primaria, en el 35,6\% de la muestra; desde la revisión por el médico de atención primaria hasta la valoración por especialista, en el 52,I \%; y desde el diagnóstico hasta el inicio de tratamiento, de $37,7 \%$ (tabla 3 ).

El 9,8 \% de los pacientes acudió a la acción judicial de tutela para recibir atención, 7I \% de las cuales fueron para la atención integral de la enfermedad. Ningún paciente rechazó el tratamiento por miedo a las secuelas funcionales o estéticas, y solo uno se demoró en tomar la decisión.

Los resultados del análisis univariado entre los factores demográficos y los clínicos, y el retraso desde el inicio de los síntomas hasta la primera consulta con el médico de atención primaria, se muestran en la tabla 4. De estos, fueron estadísticamente significativos en el análisis multivariado, el lugar de residencia rural $\left(\mathrm{OR}=4,68 ; \mathrm{IC}_{95 \%} \mathrm{I}, 09-20, \mathrm{I} 7\right)$ y la gravedad percibida como poca o nula $\left(\mathrm{OR}=5,7 ; \mathrm{IC}_{95} \% 2, \mathrm{O}-\mathrm{I} 6,5\right)$, y muy
Tabla 1. Características de los pacientes incluidos

\begin{tabular}{|c|c|c|}
\hline Variable & $\mathbf{n}$ & $\%$ \\
\hline \multicolumn{3}{|l|}{ Sexo } \\
\hline Masculino & 94 & 64,4 \\
\hline Femenino & 52 & 35,6 \\
\hline \multicolumn{3}{|l|}{ Estado civil } \\
\hline Casado & 67 & 45,89 \\
\hline Soltero & 43 & 29,45 \\
\hline Viudo & 17 & 11,64 \\
\hline Unión libre & 19 & 13,01 \\
\hline \multicolumn{3}{|l|}{ Nivel socioeconómico } \\
\hline Bajo & 30 & 20,5 \\
\hline Medio & 114 & 78,1 \\
\hline Alto & 2 & 1,4 \\
\hline \multicolumn{3}{|l|}{ Ocupación } \\
\hline Empleado & 39 & 26,7 \\
\hline Desempleado & 40 & 27,4 \\
\hline Independiente & 32 & 21,9 \\
\hline Pensionado & 35 & 24,0 \\
\hline \multicolumn{3}{|l|}{ Escolaridad } \\
\hline Primaria & 46 & 31,5 \\
\hline Secundaria & 69 & 47,3 \\
\hline Universidad & 14 & 9,6 \\
\hline Técnico & 11 & 7,5 \\
\hline Ninguno & 6 & 4,1 \\
\hline Seguridad social (contributiva) & 124 & 84,9 \\
\hline Vivienda urbana & 130 & 89,0 \\
\hline Tabaquismo & 100 & 68,5 \\
\hline Alcoholismo & 36 & 25,0 \\
\hline \multicolumn{3}{|l|}{ Localización del tumor } \\
\hline Laringe & 56 & 38,36 \\
\hline Cavidad oral & 32 & 21,92 \\
\hline Orofaringe & 29 & 19,86 \\
\hline Glándula salivar & 12 & 8,22 \\
\hline Senos paranasales & 10 & 6,85 \\
\hline Labio & 6 & 4,11 \\
\hline Otro & 1 & 0,68 \\
\hline \multicolumn{3}{|l|}{ Estadio TNM } \\
\hline I & 25 & 17,1 \\
\hline II & 34 & 23,3 \\
\hline III & 46 & 31,5 \\
\hline IV & 41 & 28,1 \\
\hline \multicolumn{3}{|l|}{ Tratamiento recibido } \\
\hline Cirugía & 72 & 49,32 \\
\hline Quimioterapia & 39 & 26,71 \\
\hline Quimioterapia más radioterapia & 13 & 8,90 \\
\hline Radioterapia & 6 & 4,11 \\
\hline Cirugía más adyuvancia & 6 & 4,11 \\
\hline Paliación & 5 & 3,42 \\
\hline Sin tratamiento & 4 & 3,42 \\
\hline
\end{tabular}


Tabla 2. Características de los síntomas y razones para no consultar

\begin{tabular}{|c|c|c|}
\hline Variable & $\mathbf{n}$ & $\%$ \\
\hline \multicolumn{3}{|l|}{ Primer síntoma } \\
\hline Masa & 56 & 38,36 \\
\hline Disfonía & 36 & 24,66 \\
\hline Odinofagia & 14 & 9,59 \\
\hline Úlcera & 13 & 8,90 \\
\hline Tos & 11 & 7,53 \\
\hline Hemoptisis & 7 & 4,79 \\
\hline Disfagia & 4 & 2,74 \\
\hline Pérdida peso & 2 & 1,37 \\
\hline Otra & 3 & 2,05 \\
\hline \multicolumn{3}{|c|}{ Causa para no buscar atención médica inmediata } \\
\hline $\begin{array}{l}\text { Pensó que el síntoma desaparecería } \\
\text { espontáneamente }\end{array}$ & 44 & 65,67 \\
\hline No sabía dónde ir & 2 & 2,99 \\
\hline Asuntos de trabajo & 1 & 1,49 \\
\hline No le dio importancia & 6 & 8,96 \\
\hline Miedo & 5 & 7,46 \\
\hline No sabe & 9 & 13,43 \\
\hline \multicolumn{3}{|l|}{ Gravedad percibida del síntoma } \\
\hline Nada & 58 & 39,73 \\
\hline Poco & 35 & 23,97 \\
\hline Moderado & 38 & 26,03 \\
\hline Grave & 10 & 6,85 \\
\hline Muy grave & 4 & 2,74 \\
\hline No sabe & 1 & 0,68 \\
\hline $\begin{array}{l}\text { Tratamiento casero antes de consultar } \\
\text { al médico de atención primaria }\end{array}$ & 7 & 4,8 \\
\hline $\begin{array}{l}\text { Tratamiento casero y posterior } \\
\text { atención por médico de familia }\end{array}$ & 10 & 8,2 \\
\hline \multicolumn{3}{|l|}{ Razones para el tratamiento casero } \\
\hline No confía en la medicina & 4 & 44,4 \\
\hline No tiene acceso & 2 & 22,2 \\
\hline No tiene tiempo & 3 & 33,3 \\
\hline No responde & 1 & 0,1 \\
\hline
\end{tabular}

Tabla 3. Distribución de tiempos de atención

\begin{tabular}{lccc}
\hline \multirow{1}{*}{ Variable } & \multicolumn{3}{c}{ Tiempo } \\
\cline { 2 - 4 } & $\begin{array}{c}<1 \text { mes } \\
\mathbf{n}(\%)\end{array}$ & $\begin{array}{c}\mathbf{1 - 2} \text { meses } \\
\mathbf{n}(\%)\end{array}$ & $\begin{array}{c}>\mathbf{2} \text { meses } \\
\mathbf{n}(\%)\end{array}$ \\
\hline $\begin{array}{l}\text { Entre aparición de sínto- } \\
\text { mas y consulta al médico } \\
\text { de atención primaria }\end{array}$ & $64(43,8)$ & $30(20,5)$ & $52(35,6)$ \\
$\begin{array}{l}\text { Entre valoración de médi- } \\
\text { co de atención primaria y } \\
\text { definición del diagnóstico }\end{array}$ & $24(16,4)$ & $49(33,6)$ & $73(50)$ \\
$\begin{array}{l}\text { Entre valoración por mé- } \\
\text { dico general y valoración } \\
\text { por especialista }\end{array}$ & $26(17,8)$ & $44(30,1)$ & $76(52,1)$ \\
$\begin{array}{l}\text { Entre el diagnóstico y el } \\
\text { inicio del tratamiento }\end{array}$ & $11(7,5)$ & $80(54,8)$ & $55(37,7)$ \\
\hline
\end{tabular}

Tabla 4. Análisis univariado del retraso entre el inicio de síntomas y la consulta a un médico de atención primaria

\begin{tabular}{lcc}
\hline Variable & OR (IC95\%) & p \\
\hline Sexo (masculino) & $1,07(0,52-2,16)$ & 0,851 \\
Vive en pareja (no) & $0,72(0,36-1,43)$ & 0,35 \\
Estrato socioeconómico (bajo) & $1,05(0,46-2,41)$ & 0,89 \\
Ocupación (activo) & $0,85(0,43-1,68)$ & 0,65 \\
Escolaridad (no o primaria) & $0,54(0,26-1,12)$ & 0,10 \\
Régimen (subsidiado) & $0,48(0,147-1,34)$ & 0,17 \\
Lugar de residencia (rural) & $3,49(1,22-9,88)$ & 0,01 \\
Masa & $0,52(0,25-1,07)$ & 0,07 \\
Disfonía & $1,91(0,89-4,1)$ & 0,09 \\
Odinofagia & $1,93(0,66-5,6)$ & 0,23 \\
Tos & $2,32(0,71-7,57)$ & 0,17 \\
Úlcera & $0,51(0,14-1,83)$ & 0,32 \\
Percepción de gravedad & $6,16(2,56-14,7)$ & $>0,001$ \\
En cavidad oral & $0,93(0,41-2,10)$ & 0,86 \\
En orofaringe & $1,13(0,49-2,59)$ & 0,77 \\
En laringe & $1,66(0,83-3,30)$ & 0,14 \\
\hline
\end{tabular}

cercanos a la diferencia estadística, la escolaridad hasta la primaria $\left(\mathrm{OR}=\mathrm{O}, 4 \mathrm{I} ; \mathrm{IC}_{95 \%} \mathrm{O}, \mathrm{I6}-\mathrm{I}, \mathrm{O} 7\right)$.

Los resultados del análisis univariado entre los factores demográficos y clínicos, y el retraso desde la primera consulta con el médico de atención primaria hasta la consulta con el especialista, se muestra en la tabla 5. De estos, fueron estadísticamente significativos en el análisis multivariado, el tipo subsidiado de seguridad social $\left(\mathrm{OR}=5,5 ; \mathrm{IC}_{95 \%} \mathrm{I}, 58-\mathrm{I} 9,42\right)$, la gravedad percibida como poca o nula $\left(\mathrm{OR}=2,45 ; \mathrm{IC}_{95 \%} \mathrm{I}, \mathrm{OI}-5,93\right)$ y muy cercana a la diferencia estadística, no tener ocupación $\left(\mathrm{OR}=2, \mathrm{I} 7 ; \mathrm{IC}_{95 \%} \mathrm{O}, 97-4,85\right)$.

Los resultados del análisis univariado entre los factores demográficos y clínicos, y el retraso entre el diagnóstico y el inicio de tratamiento, se muestran en la tabla 6. De estos, fueron estadísticamente significativos en el análisis multivariado, el tipo de seguridad social $(\mathrm{OR}=7,9$; $\mathrm{IC}_{95 \%}$ 2,2-28,3), tener tos como síntoma ( $\mathrm{OR}=\mathrm{O}, \mathrm{O} 7$; $\left.\mathrm{IC}_{95} \% \mathrm{O}, 008-0,57\right)$ y la localización en orofaringe $\left(\mathrm{OR}=4,8 ; \mathrm{IC}_{95 \%} \mathrm{I}, 4-\mathrm{I} 6,6\right)$; muy cercana a la diferencia estadística tener como síntoma disfonía (OR=0,27; IC $95 \%$ O,O7-I,I3). 
Tabla 5. Análisis univariado del retraso entre la primera consulta con médico de familia y la consulta a especialista oncológico.

\begin{tabular}{lcc}
\hline Variable & OR (IC95\%) & Valor de P \\
\hline Sexo (masculino) & $1,0(0,51-1,97)$ & 0,98 \\
Vive en pareja (no) & $0,72(0,37-1,41)$ & 0,35 \\
Estrato socioeconómico (bajo) & $1,5(0,67-3,34)$ & 0,32 \\
Ocupación (activo) & $1,39(0,73-2,67)$ & 0,31 \\
Escolaridad (no/primaria) & $0,88(0,44-1,72)$ & 0,71 \\
Régimen (subsidiado) & $3,74(1,34-10,4)$ & 0,01 \\
Lugar de residencia (rural) & $1,61(0,57-4,53)$ & 0,37 \\
Síntoma (Masa) & $0,51(0,28-1,07)$ & 0,07 \\
Síntoma (Ronquera) & $1,2(0,57-2,54)$ & 0,62 \\
Síntoma (Odinofagia) & $1,74(0,57-5,23)$ & 0,33 \\
Síntoma (Tos) & $1,14(0,34-3,61)$ & 0,86 \\
Síntoma (Ulcera) & $2,21(0,68-7,11)$ & 0,19 \\
Percepción de gravedad & $2,20(1,11-4,37)$ & 0,02 \\
Sitio de tumor (Cavidad oral) & $0,65(0,29-1,42)$ & 0,28 \\
Sitio de tumor (Orofaringe) & $0,82(0,37-1,84)$ & 0,64 \\
Sitio de tumor (Laringe) & $1,39(0,71-2,71)$ & 0,33 \\
\hline & &
\end{tabular}

Tabla 6. Análisis univariado del retraso entre el diagnóstico y el inicio de tratamiento.

\begin{tabular}{lcc}
\hline Variable & OR (IC95\%) & $\begin{array}{c}\text { Valor } \\
\text { de P }\end{array}$ \\
\hline Sexo (Masc) & $1,07(0,53-2,16)$ & 0,83 \\
Vive en pareja (no) & $0,66(0,33-1,30)$ & 0,23 \\
Estrato socioeconómico (bajo) & $1,34(0,60-3,01)$ & 0,47 \\
Ocupación (activo) & $1,03(0,52-2,0)$ & 0,93 \\
Escolaridad (no/primaria) & $1,19(0,59-2,38)$ & 0,61 \\
Régimen (subsidiado) & $4,5(1,73-11,6)$ & 0,001 \\
Lugar de residencia (rural) & $1,32(0,48-3,68)$ & 0,59 \\
Síntoma (masa) & $0,77(0,38-1,53)$ & 0,46 \\
Síntoma (ronquera) & $1,07(0,49-2,30)$ & 0,86 \\
Síntoma (odinofagia) & $0,91(0,30-2,75)$ & 0,87 \\
Síntoma (tos) & $0,34(0-1,47)$ & 0,16 \\
Síntoma (ulcera) & $1,46(0,48-4,42)$ & 0,50 \\
Percepción de gravedad & $1,13(0,56-2,26)$ & 0,73 \\
Sito de tumor (cavidad oral) & $0,38(0,15-0,94)$ & 0,03 \\
Sitio de tumor (orofaringe) & $2,4(1,08-5,56)$ & 0,02 \\
Sitio de tumor (laringe) & $1,42(0,72-2,82)$ & 0,30 \\
\hline & &
\end{tabular}

Se encontró asociación entre el retraso para la atención por especialista $\left(\mathrm{OR}=6,0 ; \mathrm{IC}_{95} \% \mathrm{I}, 45-22,7\right)$ $y$ el inicio del tratamiento $\left(\mathrm{OR}=4,8 ; \mathrm{IC}_{95} \mathrm{I}, 5-\mathrm{I} 5,4\right)$ con el uso de la acción de tutela. También, se encontró asociación estadísticamente significativa entre el estadio avanzado y el retraso para la primera atención $\left(\mathrm{OR}=2, \mathrm{I} 7 ; \mathrm{IC}_{95 \%} \mathrm{I}, 03-4,55\right)$ y un resultado cercano a la diferencia estadística con el inicio del tratamiento $\left(\mathrm{OR}=\mathrm{I}, 9 ; \mathrm{IC}_{95 \%} \mathrm{O}, 9 \mathrm{I}-3,9\right)$.

\section{Discusión}

El tratamiento del cáncer de cabeza y cuello representa un importante desafío para los profesionales del área de la salud, por las implicaciones que tiene en la expectativa de vida, la funcionalidad, las repercusiones estéticas y los costos para el sistema de salud, entre otros factores ${ }^{7}$. En Colombia, el diagnóstico de los tumores de cabeza y cuello es tardío. Esto aumenta la mortalidad, pues esta se ve directamente influenciada por el estadio, pasando de tener una supervivencia de 70 a $90 \%$ a cinco años en los estadios I y II, a una de 20 a $55 \%$ en los estadios avanzados ${ }^{8}$.

La importancia de identificar los factores clínicos y administrativos que pueden retrasar el diagnóstico y tratamiento oportunos de los pacientes con cáncer de cabeza y cuello, radica en que pueden servir de guía para futuras intervenciones con impacto directo en diversos factores, como el aumento de la supervivencia, la disminución de la morbilidad y una menor frecuencia de secuelas funcionales y estéticas 9 .

Se encontró una frecuencia alta de consumo de tabaco, comparable con lo reportado en otros estudios, pero una baja frecuencia de consumo de alcohol. El $60 \%$ de los pacientes consultaron en estadios avanzados de la enfermedad. Ni la localización del tumor, ni el nivel educativo se asociaron con el retraso en la primera visita al médico, en la valoración especializada o en el tratamiento.

El síntoma inicial de presentación se asoció con la decisión de buscar atención inmediata. El $53 \%$ de los pacientes no buscó atención inmediata porque consideraban que el síntoma 
desaparecería sin necesidad de atención médica. Se encontró que, entre los síntomas típicos de presentación existen algunos que alarman al paciente, como la hemoptisis, la masa o la pérdida de peso, y obligan a buscar atención médica oportuna. Otros síntomas, como tos, odinofagia y disfagia, son percibidos como de poco riesgo. Esto concuerda con que el $63 \%$ de los pacientes pensaba que la enfermedad era de poco riesgo (nada grave o poco grave). Se ha encontrado que los pacientes con lesiones ubicadas en áreas de poca visibilidad, como la orofaringe, suelen consultar más tardíamente ${ }^{\mathrm{IO}}$.

Es posible sugerir que el retraso entre la presentación del primer síntoma y la primera visita al médico, se debe a la falta de educación sobre signos de alarma. Stefanuto, et al. " ${ }^{\text {II }}$ encontraron que el tiempo que transcurre entre el inicio de los síntomas y la primera consulta, es el mayor responsable del retraso en el inicio de atención y el tratamiento de los pacientes con cáncer oral, con una media de 3,5 a 5,5 meses, seguido por el retraso por parte del personal de salud. Esto refuerza la idea de que parte de la estrategia de atención de los tumores de cabeza y cuello implica la educación sobre signos de alarma y la tamización en población de alto riesgo, como personas de edad avanzada y aquellas con antecedentes de tabaquismo o consumo de alcohol ${ }^{12-14}$. El Plan Nacional para el Control del Cáncer en Colombia 20I2 -202I no contempla estrategias para la detección temprana de tumores de la vía aerodigestiva, aunque sí están bien estructurados para otros tipos de tumores, como los de cuello uterino, mama, colon y estómago ${ }^{15}$.

Otro punto interesante que suele discutirse es que, antes de consultar, los pacientes intentan tratamientos alternativos. En este estudio, solo el $8 \%$ manifestó un retraso en su consulta inicial debido a la práctica de algún tratamiento no convencional. Esto contrasta con otros estudios, en los cuales hasta el 5I \% utilizó alguna sustancia medicinal alternativa en algún momento de su enfermedad ${ }^{16}$.

En este estudio, los factores que mostraron estar implicados en el retraso entre la primera consulta por el médico general y la valoración por el especialista, son el pertenecer al régimen subsidiado y tener una percepción poco grave o nada grave del síntoma inicial. Esto concuerda con lo informado por Davis, et al. ${ }^{16}$, quienes encontraron que más de la mitad de los pacientes pensaba que los síntomas mejorarían por sí mismos y solo el i3 \% tuvo una percepción de gravedad de los síntomas iniciales. Igualmente, Tromp, et al. ${ }^{17,18}$, encontraron que solo el $4 \%$ de los pacientes interpretó que sus síntomas iniciales estuvieran relacionados con cáncer.

Se sabe que el acceso al sistema de salud es determinante en el retraso en la consulta médica. Osazuwa, et al. ${ }^{13}$, encontraron que los pacientes sin seguridad social tienen un riesgo io veces mayor a presentarse en estadios avanzados de la enfermedad. En este estudio, los pacientes que pertenecían al régimen subsidiado presentaron un retraso entre la consulta al médico de atención primaria y la valoración especializada, y entre el tiempo de diagnóstico y el inicio del tratamiento. Cariati, et al. ${ }^{\mathrm{I}}$, encontraron tiempos de espera prolongados que pueden llegar ser tan largos como 24 a 48 semanas. Esto puede atribuirse a dificultades locales administrativas y de acceso.

El Io \% de los pacientes interpusieron una acción legal para recibir atención. No fue posible establecer una asociación entre el tipo de aseguradora y el retraso en la atención, el diagnóstico y el tratamiento de los pacientes con tumores de la vía aerodigestiva. Sin embargo, existe una percepción negativa sobre la calidad del sistema de salud colombiano. Según datos del Ministerio de Salud de Colombia, el $42 \%$ de las personas no consigue una cita con un médico de atención primaria en los primeros tres días y, de estas, al $4 \%$ les toma más de un mes el conseguirla. El $43 \%$ de los pacientes en Colombia se demora más de $\mathrm{I} 5$ días en obtener una cita con un médico especialista y, de estos, el $19 \%$ demora más de un mes ${ }^{19}$. Las debilidades en la gestión por parte del asegurador pueden explicar esta gran frecuencia del uso de los recursos legales. Vivir en el área rural también se 
asoció con retraso entre la aparición del síntoma y la primera valoración por médico de atención primaria. En áreas rurales de Colombia, los niveles de pobreza son cercanos al $65 \%$, por lo que las condiciones socioeconómicas o la vivienda en regiones aisladas pueden ser un obstáculo para acceder a una atención oportuna.

Existen algunas debilidades en este estudio. El sesgo de memoria puede influenciar los datos provistos por los pacientes. No se exploraron las causas del retraso entre la consulta al médico de atención primaria y la consulta especializada. Esta puede retrasarse por falta de conocimiento del médico de atención primaria para identificar precozmente los signos que indican una enfermedad maligna o por trámites administrativos. Finalmente, aunque se interrogó sobre el estado civil, no se exploró la situación de vivir en soledad. Este factor se ha asociado a retraso en la consulta y en el tratamiento ${ }^{20}$.

\section{Conclusión}

Más de la mitad de los pacientes con cáncer de cabeza y cuello se presenta en estadios avanzados de la enfermedad y esto se explica por un retraso en la atención medica específica. Algunos síntomas de presentación, la percepción de gravedad del síntoma, el lugar de procedencia y el tipo de seguridad social, son factores asociados con el retraso. En cualquier estrategia para disminuir el retraso, se deben tener en cuenta estos factores.

\section{Cumplimiento de normas éticas}

Conflicto de interés: ninguno

Consentimiento informado: este estudio fue aprobado con el Comité de Ética en Investigación de la Fundación Colombiana de Cancerología. De acuerdo con la Resolución 008430 de 1993 del Ministerio de Salud de Colombia, se consideró que la investigación no tenía riesgo para los pacientes, por lo tanto, no se diligenció el consentimiento informado.

Fuentes de financiación: recursos propios de los autores

\section{Referencias}

I. Lambert R, Sauvaget C, de Camargo-Cancela M, Sankaranarayanan R. Epidemiology of cancer from the oral cavity and oropharynx. Eur J Gastroenterol Hepatol. 20II;23:633-4I.

2. Pardo C, Cendales R. Incidencia, mortalidad y prevalencia de cáncer en Colombia, 2007-20II. Bogota: Instituto Nacional de Cancerologia; 2015.

3. Ang KK, Harris J, Wheeler R, Weber R, Rosenthal DI, Nguyen-Tan PF, et al. Human papillomavirus and survival of patients with oropharyngeal cancer. $\mathrm{N}$ Engl J Med. 20I0;363:24-35.

4. D'Souza G, Kreimer AR, Viscidi R, Pawlita M, Fakhry C, Koch WM, et al. Case-control study of human papillomavirus and oropharyngeal cancer. N Engl J Med. 2007;356:1944-56.

5. Ferreiro J, García JL, Barceló R, Rubio I. Quimioterapia: efectos secundarios. Gaceta Médica de Bilbao. 2003;I00:69-74.

6. Departamento Administraivo Nacional de Estadística (DANE). Pobreza multidimensional en Colombia. Boletin Técnico. Bogotá: Departamento Administrativo Nacional de Estadistica; 208. p. 24.

7. van der Waal I, de Bree R, Brakenhoff R, Coebergh JW. Early diagnosis in primary oral cancer: Is it possible? Med Oral Patol Oral Cir Bucal. 20II;16:e300-5.

8. Gómez I, Warnakulasuriya S, Varela-Centelles PI, López-Jornet P, Suárez-Cunqueiro M, Diz-Dios P, et al. Is early diagnosis of oral cancer a feasible objective? Who is to blame for diagnostic delay? Oral Dis. 20I0;I6:333-42.

9. Seoane J, Takkouche B, Varela-Centelles P, Tomas I, Seoane-Romero JM. Impact of delay in diagnosis on survival to head and neck carcinomas: A systematic review with meta-analysis. Clin Otolaryngol. 20I2;37:99-IO6.

Io. Noonan B. Understanding the reasons why patients delay seeking treatment for oral cancer symptoms from a primary health care professional: An integrative literature review. Eur J Oncol Nurs. 20I4;I8:II8-24.

II. Stefanuto P, Doucet JC, Robertson C. Delays in treatment of oral cancer: A review of the current literature. Oral Surg Oral Med Oral Pathol Oral Radiol. 20I4;II7:424-9.

I2. Guneri P, Epstein JB. Late stage diagnosis of oral cancer: components and possible solutions. Oral Oncol. 20I4;50:II3I-6.

I3. Osazuwa-Peters N, Christopher KM, Hussaini AS, Behera A, Walker RJ, Varvares MA. Predictors of stage at presentation and outcomes of head and neck cancers in a university hospital setting. Head Neck. 2016;38(Suppl.I):EI826-32.

I4. Cariati P, Pérez de Perceval Tara M, Fernández J, Martínez I. [Factors that influence the diagnostic delay of oral cancer]. Semergen. 20I8;44:eI05-6. doi: Io.IOI6/j. semerg.2018.03.003 
I5. Instituto Nacional de Cancerología. Plan nacional para el control de cáncer en Colombia 20I2-2020. Bogota: Ministerio de Salud y Proteccion Social; 2012.

I6. Davis GE, Bryson CL, Yueh B, McDonell MB, Micek MA, Fihn SD. Treatment delay associated with alternative medicine use among veterans with head and neck cancer. Head Neck. 2006;28:926-3I.

I7. Tromp DM, Brouha XD, De Leeuw JR, Hordijk GJ, Winnubst JA. Psychological factors and patient delay in patients with head and neck cancer. Eur J Cancer. 2004;40:I509-I6.

I8. Tromp DM, Brouha XD, Hordijk GJ, Winnubst JA, de Leeuw JR. Patient factors associated with delay in pri- mary care among patients with head and neck carcinoma: A case-series analysis. Fam Pract. 2005;22:554-9.

19. Delgado-Gallego ME, Vázquez-Navarrete ML, de Moraes-Vanderlei L. [Quality of health-care according to social actors' frameworks of meaning in Colombia and Brazil]. Rev Salud Pública (Bogotá). 20I0;I2:533-45.

20. Osazuwa-Peters N, Christopher KM, Cass LM, Massa ST, Hussaini AS, Behera A, et al. What's love got to do with it? Marital status and survival of head and neck cancer. Eur J Cancer Care (Engl). 2019;28: eI3022. doi: IO.IIII/ecc.I3O22. 